\section{Leukoplakia of the Bladder}

Leukoplakia of the bladder, whether alone or associated with similar changes in the upper urinary tract, would appear to be rare enough to warrant comment whenever a case arises.

\section{CASE Report}

A man aged 59 was admitted to this hospital in April, 1950 , with severe haematuria. He was anaemic-looking and had a blood pressure of $160 / 80 \mathrm{~mm}$. $\mathrm{Hg}$. The bladder was not palpable abdominally, the kidneys did not appear clinically to be enlarged, and rectal examination suggested no abnormality of prostate or seminal vesicles. Neither was any abnormality found in other systems. A provisional diagnosis of vesical neoplasm was made. As his haemoglobin was only $53 \%$ a blood transfusion was given, and this was repeated twice in the subsequent few days.

His Wassermann reaction was negative, and intravenous pyelography showed the kidneys to be normal. Ten days
Low-power view of bladder wall. $(\times 114$.

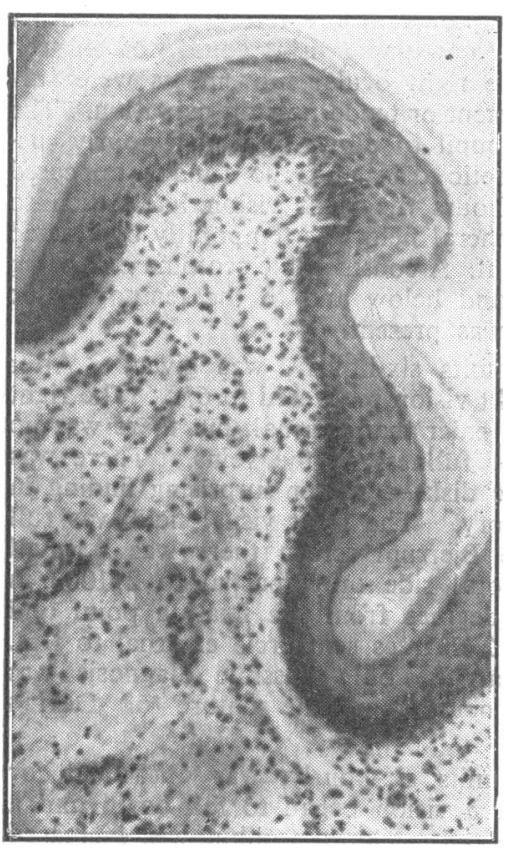
after admission he developed clot retention, and suprapubic cystotomy was performed by one of us (L.M.Z.). After evacuation of many clots no evidence of vesical neoplasm could be found and a clear efflux was observed from both ureteric orifices. The bladder mucous membrane had a peculiar white appearance and was very smooth. A biopsy of the bladder wall was taken and the bladder closed round a suprapubic tube. The histological report stated: "The covering epi thelium is com posed entirely of

keratinizing epidermoid type with eleidin granules. There is subacute inflammation in the subjacent connective tissue, and beneath this is smooth muscle. The epithelium is remarkable if it comes from the bladder, as it does not at all resemble normal vesical epithelium." (See Figure.) As it did in fact come from the bladder a diagnosis of leukoplakia was made. After operation the bleeding slowly subsided and the suprapubic fistula was allowed to heal. The patient was discharged 27 days after operation, passing clear urine quite normally.

He remained symptom-free until November, 1952, when he was again admitted with fairly severe haematuria. This persisted, and as cystoscopy was unsatisfactory and it was feared that a squamous carcinoma might now have developed, the bladder was again opened. The state of affairs was exactly as before and no growth could be seen. Histological examination of a further biopsy merely confirmed the previous findings. Again the bleeding settled after the cystotomy, and he was discharged after 26 days.

Since 1952 he has had very little haemorrhage, but the suprapubic scar has broken down three times, and there has been leakage of urine. Each time, however, it has healed readily after urethral bouginage and antibiotic treatment.

Cystoscopy was last performed in December, 1954, and showed a whitish bladder mucosa with much fibrinous debris on its walls due to superadded infection. There was no haematuria, but the urine was rather dirty and foulsmelling, and yielded a heavy growth of Pseudomonas aeruginosa which was insensitive to all antibiotics except polymyxin. The haemoglobin was $87 \%$ and the blood urea $30 \mathrm{mg}$. per $100 \mathrm{ml}$.

\section{Discussion}

Leukoplakia of the urinary bladder was first described by Rokitansky in 1861, and has been the subject of much controversy ever since. Up to 1929 Patch could find only 123 cases in the literature, including cases where the kidneys and ureters as well as the bladder were involved. Connery (1953) found only 45 cases of proved leukoplakia of the bladder in the records of the Mayo Clinic covering the years 1915 to 1947.

Leukoplakia is a disease of undetermined aetiology involving mucous membrane. It is characterized by an epidermoid metaplasia in various stages of keratinization, and histologically the end-result closely resembles normal skin. Though the true aetiology remains obscure, most writers strongly favour chronic infection or chronic irritation as the most likely cause. Another theory is that vitamin-A deficiency may be a factor in causation, and a number of cases have been associated with a positive Wassermann reaction. It has hitherto been generally agreed that leukoplakia is a precursor of squamous carcinoma, but recent work suggests that this is incorrect, although the condition itself is still considered irreversible. Our own experience gives us no reason to suspect that the condition is precancerous.

From the cases reported it appears that leukoplakia of the bladder is three times as common in the male as in the female, and is most frequent in the fifth decade of life. The usual urinary symptoms are haematuria and dysuria, but these are probably due to the superimposed chronic cystitis. Clinically the picture may be confused by such symptoms as loss of weight, fever, nausea, and vomiting. $X$-ray examination of the kidneys and bladder usually reveals no abnormality, so that most investigators consider that diagnosis before operation is highly improbable. However, the appearance of desquamated epithelial tissue in the urine must be considered pathognomonic. The bladder appears to be the most common site of leukoplakia in the urinary tract, and the renal pelvis to be less often affected, while it is estimated that in only $10 \%$ of cases of the latter is the bladder also involved.

Various methods of treating the condition have been suggested, including massive doses of vitamin $A$ and a full course of antibiotic therapy, for which aureomycin is considered best. Winsbury-White (1932) reports a case of leukoplakia in which radium implanted into an ulcerated area at the base of the bladder led to the healing of a suprapubic fistula created during a diagnostic cystotomy. Ewert and Hoffman (1944) report good effect from instillation of sterile cod-liver oil into the bladder, and Helfert and Bremen (1953) claim apparent arrest of renal leukoplakia with bladder involvement following a course of A.C.T.H. As a preventive measure the thorough treatment of chronic urinary infection is emphasized, and the treatment of tertiary syphilis if this is also present.

Our thanks are due to Dr. A. J. McCall for the histological reports and to Dr. W. G. D. Caldwell for the photograph.

\section{M. ZINCK, M.D., F.R.C.S.Ed., Visiting Surgeon. \\ H. S. TRafFord, M.B., F.R.C.S.Ed.,}

North Staffordshire Royal Infirmary. Stoke-on-Trent.

\section{REFERENCES}

Connery, D. B. (1953). J. Urol., 69, 121

Ewert, E. E., and Hoffmar, H. A. (1944). Lahey Clin. Bull.: 4. 27. Helfert, I., and Bremen, H. A. (1953). Ohio St. med. J., 49, 109. Patch, F. S. (1929). New Engl. J. Med., 200, 423.

Winsbury-White, H. P. (1932). Brit. J. Surg., 20, 49 